\title{
Rasgos del fenómeno del cambio climático en la realidad colombiana ${ }^{1}$
}

https://doi.org/10.21830/9789585318342.08

\author{
Anderson Castro Carreño ${ }^{2}$ \\ Escuela de Aviación del Ejército \\ John Cristhian Fernández Lizarazo ${ }^{3}$ \\ Escuela de Aviación del Ejército \\ John Alexander León Torres ${ }^{4}$ \\ Escuela de Armas Combinadas del Ejército \\ Sammy Hernesto Rodríguez Lemus \\ Escuela de Ingenieros del Ejército
}

\section{Resumen}

Ante los drásticos fenómenos asociados al clima es evidente que algo está ocurriendo con nuestro planeta y Colombia está inmersa en esa transformación. De ahí la necesidad de conocer las causas de estos fenómenos climáticos, con el fin de reducir la vulnerabilidad del país e incre-

1 Este capítulo hace parte de los resultados de los proyectos de investigación "Reciclaje químico de residuos de lubricantes generados en la División de Aviación Asalto Aéreo" y "Acciones estratégicas del Ejercito Nacional orientadas al desarrollo sostenible en Colombia. Experiencia y reflexión desde la minería y los objetivos de desarrollo sostenible” del Grupo de Investigación en Aviación Militar de la Escuela de Aviación del Ejercito, registrado con el código COL0077618 y categorizado en C por Minciencias. Los puntos de vista y los resultados de este capítulo pertenecen a los autores y no reflejan necesariamente los de las instituciones participantes.

2 Magíster en Ciencias-Química (Universidad Nacional de Colombia). Especialista en Educación y Gestión Ambiental (Universidad Nacional de Colombia). Licenciado en Química (Universidad Distrital Francisco José de Caldas). Docente e investigador de los Grupos de Investigación de ESAVE-ESACE. Orcid: https://orcid.org/0000-0003-0087-8932 - Contacto: andersoncastrocarreno@cedoc.edu.co

$3 \mathrm{PhD}$ en Ciencias Agropecuarias y magister en Ciencias Agrarias (Universidad Nacional de Colombia. Licenciado en Biología (Universidad Francisco José de Caldas). Director del Programa de Ingeniería Agronómica de la Universidad de La Salle. Orcid: https://orcid.org/0000-0001-9015-7404 - Contacto: johfernandez@lasalle.edu.co

4 Teniente Coronel del Ejército Nacional de Colombia. Estudiante de la Maestría en Educación (Universidad Militar Nueva Granada). Especialización en Seguridad y Defensa Nacionales (Escuela Superior de Guerra "General Rafael Reyes Prieto"). Especialización en docencia Universitaria (Centro de Educación Militar). Profesional en Ciencias Militares (Escuela Militar de Cadetes "General José María Córdova”). Profesional en Relaciones Internacionales y Estudios Políticos (Universidad Militar Nueva Granada). Inspector de Estudios de la Escuela de Armas Combinadas del Ejército. Orcid: https://orcid.org/0000-0001-6279-8351 - Contacto: johnleontorres@cedoc.edu.co

5 Mayor del Ejército Nacional de Colombia. Especialista en Gerencia Integral de Obras (Escuela de Ingenieros Militares). Profesional en Ciencias Militares (Escuela Militar de Cadetes). Administrador de Empresas (Universidad Militar Nueva Granada). Orcid: https://orcid.org/0000-0001-5352-7349 - Contacto: sammy.rodriguez@buzonejercito.mil.co 
mentar su capacidad de respuesta frente a las amenazas e impactos del cambio climático. Como se trata de un fenómeno planetario es importante comprender la dinámica de nuestro planeta en lo que tiene que ver con la producción y acumulación de gases efecto invernadero. A partir de una revisión documental se analizaron las afectaciones del cambio climático en la geografía colombiana, en particular en los ecosistemas de páramo, por su alta vulnerabilidad a los cambios de las condiciones del clima. En ese orden de ideas, se proponen acciones para la adaptación al cambio climático y para mitigar sus efectos.

Palabras clave: clima; prevención de desastres; deterioro ambiental; efecto invernadero.

\section{Introducción}

La realidad del cambio climático global, tal y como lo afirma el Grupo Intergubernamental de Expertos sobre el Cambio Climático (en adelante IPCC) (2014), es innegable. Durante el presente siglo, ha sido notorio el incremento de la temperatura global, constituyendo el principal problema ambiental de nuestro planeta y la mayor amenaza global para el desarrollo. Las emisiones de gases de efecto invernadero (en adelante GEI) producidas por la acción humana, en particular las procedentes del consumo de petróleo, gas natural y carbón, son la causa primordial de calentamiento (IPCC, 2014).

Los impactos del calentamiento global se perciben con frecuencia como una cuestión del futuro lejano o como si solo estuviesen afectando a algunas especies de animales que habitan en el Ártico (Rodríguez \& Mance, 2009). Pero los efectos del cambio climático ya son evidentes en Colombia. Este capítulo busca evidenciar dichos impactos en el territorio colombiano y visualizar algunos esfuerzos del Ejército colombiano para la adaptación de ecosistemas estratégicos altamente afectados por este fenómeno climático.

El presente acápite da cuenta de una investigación, cualitativa-documental con características descriptivas y analíticas, realizada con el fin de conceptualizar acerca del fenómeno de cambio climático e indagar sobre programas gubernamentales relacionados con las estrategias de adaptación al cambio climático propuestos en Colombia. De acuerdo con lo anterior, las discusiones permitirán estimar una reflexión acerca de las evidencias del cambio climático en Colombia y la enunciación de estrategias de mitigación y adaptación que conduzcan a procesos efectivos de restauración de los ecosistemas de páramo y bosque andino. 


\section{Marco teórico}

\section{Realidad del cambio climático global}

Desde la década de los cincuenta, la atmósfera y el océano se han calentado, los volúmenes de nieve y hielo han disminuido, el nivel del mar se ha elevado y las concentraciones de GEI han aumentado.

Si bien es cierto que el planeta ha estado en permanente cambio y que algunos de estos cambios tienen su origen en causas naturales, como los grandes movimientos geológicos o la evolución de las especies a lo largo de la historia de la vida en la Tierra, el acelerado cambio climático tiene causas ligadas a las actividades humanas.

El IPCC afirma que la principal actividad humana que ha causado el cambio climático y que lo seguirá causando es el consumo de combustibles fósiles, en particular petróleo, carbón y gas natural, que emiten dióxido de carbono. El mecanismo mediante el cual el $\mathrm{CO}_{2}$ y otros gases producen el calentamiento global se denomina efecto invernadero (Rodríguez \& Mance, 2009).

Los GEI que se encuentran en la atmósfera absorben parte de la radiación producida por la Tierra y la envían en todas las direcciones. Entre más alta sea la concentración de GEI mayor es la captura del calor. Estos gases son: el dióxido de carbono $\left(\mathrm{CO}_{2}\right)$; el metano $\left(\mathrm{CH}_{4}\right)$; el vapor de agua; el óxido nitroso $\left(\mathrm{N}_{2} \mathrm{O}\right)$; los fluorocarbonados; los hidrofluorocarbonados; el perfloroetano y el hexafluoruro de azufre. Cada uno de los GEI tiene una capacidad diferente de atrapar el calor solar que devuelve a la Tierra en forma de radiación infrarroja.

El $\mathrm{CO}_{2}$ es el mayor causante del efecto invernadero y el que más ha contribuido al calentamiento global; se produce como consecuencia del consumo de los combustibles fósiles (petróleo, carbón y gas) y de la deforestación. Algunos GEI capturan la radiación mejor que otros, como es el caso de los fluorocarbonados, cuya concentración en la atmósfera es relativamente baja, pero cuyo poder es mayor que el de los otros GEI (Rodríguez \& Mance, 2009).

Si la atmósfera terrestre no tuviera GEI, el planeta tendría una temperatura $30{ }^{\circ} \mathrm{C}$ más fría —o de $18{ }^{\circ} \mathrm{C}$ bajo cero—, lo que lo haría inhóspito para la vida. En contraste, si su concentración fuese muchísimo más alta, la temperatura podría llegar a extremos tales que harían que la Tierra tampoco fuera factible para la vida (Rodríguez \& Mance, 2009).

La Tierra tiene la capacidad para absorber el $\mathrm{CO}_{2}$, pero esta capacidad es limitada. El problema es que, cada vez más, se deposita una mayor parte de dióxido de carbono en la atmósfera y esto ha ocasionado el aumento de la temperatura promedio del planeta. 
La interferencia humana sobre el sistema climático genera impactos observables y riesgos futuros para los sistemas humanos y naturales. Los impactos observados más significativos del cambio climático en los sistemas físicos naturales corresponden a cambios en la precipitación, alteración de los sistemas hidrológicos, deshielo, pérdida de glaciares y afectaciones en la escorrentía (Ministerio de Ambiente y Desarrollo Sostenible, 2017).

En cuanto a los sistemas biológicos, muchas especies terrestres, dulceacuícolas y marinas han cambiado su rango geográfico, actividades estacionales, patrones migratorios, abundancias e interacciones con otras especies debido al cambio climático (IPCC, 2014a). De hecho, para la región de América Central y Suramérica, el IPCC destaca tres riesgos: 1) disminución en la disponibilidad de agua en regiones semiáridas y aquellas que dependen del aporte de glaciares; 2) inundaciones; y 3) deslizamientos en áreas urbanas y rurales por el aumento de la precipitación (Ministerio de Ambiente y Desarrollo Sostenible, 2017).

\section{Importancia de escuchar a los expertos}

En la actualidad existe un consenso científico en torno a que está ocurriendo un calentamiento global que trae asociados eventos como el ascenso del nivel del mar y la ocurrencia de fenómenos naturales catastróficos asociados con las alteraciones del clima.

En 1988, al detectar el problema del cambio climático mundial, el Programa de las Naciones Unidas para el Medio Ambiente (PNUMA) y la Organización Meteorológica Mundial (OMM) crearon el IPCC con el propósito de realizar evaluaciones integrales del estado de los conocimientos científicos, técnicos y socioeconómicos sobre el cambio climático, sus causas, posibles repercusiones y las posibilidades de adaptación y respuesta al mismo.

El IPCC, en su informe de 2019, establece que:

Se estima que las actividades humanas han causado un calentamiento global de aproximadamente $1,0^{\circ} \mathrm{C}$ y es probable que el calentamiento global llegue a $1,5^{\circ} \mathrm{C}$ entre 2030 y 2052. [...] El calentamiento causado por las emisiones antropógenas durará de siglos a milenios y seguirá causando nuevos cambios a largo plazo en el sistema climático, como un aumento del nivel del mar, acompañados de impactos asociados. (IPCC, 2019, p. 7)

En cuanto a la proyección de los modelos climáticos, estos prevén aumentos de la temperatura media en la mayoría de las regiones terrestres y oceánicas, de los episodios de calor extremo en la mayoría de las regiones habitadas, de las precipi- 
taciones intensas en varias regiones, de la probabilidad de sequía y de déficits de precipitación en algunas regiones (IPCC, 2019)

De la misma manera, se prevé que para 2100 el nivel del mar seguirá aumentando. Es importante que ese aumento ocurra a menor velocidad de lo que está ocurriendo actualmente, para que haya mayor posibilidad de adaptación en los sistemas humanos y ecológicos de las islas pequeñas y las poblaciones ubicadas en zonas costeras.

En lo que tiene que ver con la superficie terrestre, con un aumento de la temperatura global, se prevén impactos en la biodiversidad y en los ecosistemas, en cuanto a pérdida y extinción de especies.

Otros preocupantes impactos están asociados a los riesgos para la salud, los medios de subsistencia, la seguridad alimentaria, el suministro de agua, la seguridad humana y el crecimiento económico (IPCC, 2019).

\section{Manifestaciones del cambio climático en Colombia}

De acuerdo con el Banco Mundial, Colombia presenta la tasa más alta en América Latina de desastres recurrentes provocados por fenómenos naturales, con más de 600 eventos reportados anualmente. También ocupa el décimo lugar dentro de los países con mayor riesgo económico a causa de desastres en el mundo, ya que el 84,7 \% de su población está localizada en áreas expuestas a dos o más peligros naturales (Rodríguez \& Mance, 2009).

Según el Departamento Nacional de Planeación (DNP-BID-Cepal, 2014), de 2010 a 2013 el número de eventos hidrometeorológicos fue de 8504, 2,6 veces más de los ocurridos de 2006 a 2009. Las familias pasaron de 4,4 millones a 6,4 millones. La vulnerabilidad del país ante los fenómenos climáticos extremos (eventos extremos de sequía y olas de lluvia) se constató durante el fenómeno de La Niña de 2010-2011, en esa ocasión el país sufrió pérdidas por más de 11 billones de pesos. En 2015, el fenómeno de El Niño causó una grave sequía y se registraron enormes pérdidas para la actividad agropecuaria (Rodríguez \& Mance, 2009; Mayorga et al., 2012).

La elaboración de escenarios de cambio climático para Colombia se ha venido realizando, desde comienzos del presente siglo, con la aplicación de la metodología de escalamiento estadístico de los productos de modelos globales de circulación general de la atmósfera (Pabón, 2012; Eslava \& Pabón, 2001; Pabón et al., 2001).

En el informe "Colombia, Primera Comunicación Nacional ante la Convención de las Naciones Unidas sobre el Cambio Climático” (2001) se reportan resultados de escenarios de cambio climático para el Valle del Cauca desarrollados 
por Mulligan (2000) y por Hulme y Sheard (1999) para los países del sector norte de la región Andina.

El Instituto de Hidrología, Meteorología y Estudios Ambientales (en adelante Ideam) ha estado realizando estudios sobre la generación de indicadores de cambio climático en Colombia, a partir de datos históricos de temperatura y precipitaciones. De acuerdo con los análisis realizados por el Ideam, en los últimos años se están presentando varios impactos en los páramos. Según Mayorga, Hurtado y Benavides (2011) los impactosasociados al cambio climático en los páramos son: fuertes incrementos de la temperatura, tendencia a la disminución de la precipitación acumulada anual y una tendencia a la disminución de eventos extremos de lluvia (aguaceros), contrario a lo que se ha evidenciado en los otros pisos térmicos.

El incremento de la temperatura en zonas de gran elevación se evidencia en el fuerte retroceso del área de los glaciares en Colombia. Al respecto, de acuerdo con Ochoa (2020), solo quedan 6 de los 19 nevados que existían en Colombia en el siglo XIX. El área glaciar del país pasó de 348 km², en 1850, a 36,6 km², en 2018. Como ejemplo de esta situación, el autor reporta la disminución de la nieve en el Volcán Nevado de Santa Isabel, ubicado entre los departamentos de Risaralda, Caldas y Tolima, la cual pasó de $27,8 \mathrm{~km}^{2}$, que lo conformaban en 1850, a 0,63 $\mathrm{km}^{2}$, distribuidos en ocho pequeños pedazos de hielo cenizo y nieve que poco a poco se derriten (Ochoa, 2020).

Es importante recalcar la importancia de los páramos como principales fuentes abastecedoras de agua para las grandes ciudades, ya que allí nacen gran parte de las quebradas y ríos del país. La disminución de las precipitaciones y la tendencia de aumento de las temperaturas durante el día provocan una mayor evaporación del agua en estas zonas, pues son factores que alteran esa importante función ecológica.

Otro ecosistema con alto impacto ambiental negativo es la selva húmeda tropical, la cual también tiene pronósticos preocupantes frente al cambio climático. La degradación amazónica tiene efectos globales por su regulación del vapor de agua y de emisiones de dióxido de carbono y por el sinnúmero de especies que habitan en ella. Rodríguez y Mance (2009) afirman que el calentamiento global afectará los ciclos de lluvia en la región amazónica, lo que puede provocar un aumento de la evapotranspiración de las plantas y una disminución de la cantidad del agua del suelo selvático.

\section{Cambio climático en Colombia: adaptación y mitigación}

Como respuesta a los evidentes impactos del cambio climático sobre los ecosistemas y la biodiversidad, ante la ocurrencia de fenómenos climáticos extremos y 
ante el riesgo de afectaciones a la producción de alimentos, en Colombia se formuló, en 2017, la Política Nacional de Cambio Climático, que busca articular esfuerzos y programas tales como la Estrategia Colombiana de Desarrollo Bajo en carbono (ECDBC), el Plan Nacional de Adaptación al Cambio Climático (PNACC), y la Estrategia Nacional REDD+, entre otras iniciativas que aportan elementos para orientar estratégicamente todos los esfuerzos hacia el cumplimiento del compromiso adquirido en el marco del Acuerdo de París (Ministerio de Ambiente y Desarrollo Sostenible, 2017) (Murillo et al., 2017).

Frente a la adaptación al cambio climático, el Ministerio de Ambiente y Desarrollo Sostenible plantea un conjunto de procesos que buscan maximizar el rol de los socioecosistemas en la reducción de los riesgos climáticos y el aumento de la resiliencia del país frente a condiciones de variabilidad y cambio climático (Ministerio de Ambiente y Desarrollo Sostenible, 2018).

Los procesos se basan en el aprovechamiento de la gran cantidad de ecosistemas estratégicos que posee Colombia, los cuales ofrecen una gama amplia de bienes y servicios que se relacionan directamente con el clima. Tal es el caso de la regulación hídrica y climática, en interacción constante con variables meteorológicas como el viento, la radiación solar o la composición del suelo, que contribuyen a la seguridad alimentaria, proveen medios de subsistencia, determinan modos de vida y ayudan a la prevención de riesgos, principalmente los asociados con procesos erosivos como los deslizamientos e inundaciones (Ministerio de Ambiente y Desarrollo Sostenible, 2018).

Por su parte, el Ejército Nacional de Colombia, en su Plan Estratégico Militar 2030, plantea como objetivos contribuir al desarrollo sostenible del país y a la protección y preservación del medioambiente. Para ello, propone las siguientes acciones: 1) implementación de viveros forestales militares; 2) restauración ecológica de páramos, mediante la propagación y siembra de frailejones; y 3) restauración ecológica del bosque alto, por medio de propagación y siembra de palma de cera (Castro et al., 2020). Para cumplir estos objetivos, desde la Dirección de Gestión Ambiental del Ejército, se prevé la implementación de 373 viveros forestales, la reforestación del mismo número de áreas deforestadas, la protección de fuentes hídricas, la siembra de 5.600.000 árboles, la germinación de 1.000.000 de frailejones y 500 palmas de cera (Castro et al., 2020).

La mitigación del cambio climático y la adaptación que ello supone, se constituyen en un reto para responder a los impactos del cambio climático. La Política Nacional de Cambio Climático concibe la adaptación como el conjunto de acciones y medidas encaminadas a reducir la vulnerabilidad de los sistemas naturales y 
humanos ante los efectos reales o esperados del cambio climático (Ministerio de Ambiente y Desarrollo Sostenible, 2017).

Aunque Colombia y, en general, la mayoría de los países de Latinoamérica y el Caribe tienen una baja participación en emisiones de GEI, presentan una alta vulnerabilidad a los efectos del cambio climático, debido a sus características geográficas, ecológicas y socioeconómicas, lo que hace indispensable la adaptación como una respuesta a los efectos del clima sobre estos territorios (Ministerio de Ambiente y Desarrollo Sostenible, 2017; Posada, 2007).

En materia de cambio climático, Colombia tiene como meta para el año 2030 reducir en un $20 \%$ las emisiones de carbono, y como compromisos de adaptación se ha propuesto alcanzar un $100 \%$ del territorio del país con planes integrales de cambio climático, aumentar 2,5 millones de hectáreas de áreas protegidas y lograr la delimitación y protección de 36 páramos, entre otros (DNP, 2018).

Frente al reto de la adaptación y mitigación al cambio climático, Rodríguez y Mance (2009) proponen las siguientes medidas:

Adaptación basada en los ecosistemas, por su capacidad de protección de la manifestación de fenómenos climáticos extremos en conjunto con las comunidades asociadas a estos ecosistemas. Los autores plantean como ejemplo las cuencas hidrográficas, que actúan como barreras naturales durante épocas de fuertes lluvias y suministran agua durante épocas de escasez; o los manglares, que actúan como zonas amortiguadoras contra las inundaciones.

Articulación de la adaptación al cambio climático y la gestión de riesgo a partir del estudio de las tendencias de los fenómenos de variabilidad climática (sequías y fuertes aguaceros) en cada zona, para identificar medidas locales acertadas para afrontarlos.

Adaptación de infraestructura básica y sectores productivos de acuerdo con las variaciones climáticas de las diferentes regiones del país (inundaciones, sequías, derrumbes).

\section{Conclusiones}

Tanto el cambio climático global como sus impactos sobre ecosistemas estratégicos de nuestro país son hechos innegables, los cuales exigen de todos asumir retos específicos desde nuestros roles particulares en la sociedad. No se trata de generar alarmas, sino de identificar alternativas de intervención.

Una alternativa importante es la de resguardar las áreas protegidas, donde se encuentran muchos de los ecosistemas que se constituyen en el principal escudo 
contra los graves efectos del cambio climático. Las acciones de restauración ecológica de zonas de bosque alto andino y de páramo, previstas desde el Ejército Nacional de Colombia, aportan enormemente a los procesos de protección de estos ecosistemas estratégicos.

De igual manera, el enfoque de adaptación al cambio climático basado en ecosistemas, desarrollado por el Ministerio de Ambiente y Desarrollo Sostenible, brinda herramientas a los habitantes de las regiones más vulnerables para adaptarse a los efectos adversos del cambio climático, a partir del uso de la biodiversidad y los servicios de los ecosistemas, como parte de una estrategia global de adaptación.

\section{Referencias}

Castro, A., Parra, M., León, J., \& Arango, I. (2020). Memorias. Primer encuentro de desarrollo y sostenibilidad ambiental. Escuela de Armas Combinadas del Ejército; Escuela de Aviación del Ejército.

DNP - Departamento Nacional de Planeación. (2017). El país debe incrementar sus inversiones en mitigación en 2,3 billones de pesos anuales para cumplir la meta de París: DNP. Portal Web DNP. Tomado de: https://bit.ly/3hanhN7

DNP - Departamento Nacional de Planeación. (2018). https://twitter.com/DNP_Colombia/ status/1063431991763312641/photo/1

Eslava, J., \& Pabón, J. (2001). Proyecto "Proyecciones climáticas e impactos socioeconómicos del cambio climático en Colombia”. Meteorología Colombiana, (3), 1-8.

Hulme, M., \& Sheard, N. (1999). Escenarios de cambio climático para países de los Andes del norte, unidad de investigación climática. University of East Anglia.

Ideam. (2001). Colombia, Primera Comunicación Nacional ante la Convención de las Naciones Unidas sobre el Cambio Climático. Ideam.

IPCC. (2014). Assessment Reports. IPCC. http://www.ipcc.ch/publications_and_data/publications_ and_data_reports.shtml

IPCC. (2014a). Summary for policymakers. En C. Field, V. Barros, D. Dokken, K. Mach, M. Mastrandrea, T. Bilir, M. Chatterjee, K. Ebi, Y. Estrada, R. Genova, B. Girma, E. Kissel, A. Levy, S. MacCracken, P. Mastrandrea, \& L. White (Eds.), Climate Change 2014: Impacts, Adaptation and Vulnerability (pp. 1-32.). Cambridge University Press.

IPCC. (2019). Grupo Intergubernamental de Expertos sobre el Cambio Climático. IPCC. https:// www.ipcc.ch/site/assets/uploads/sites/2/2019/09/IPCC-Special-Report-1.5-SPM_es.pdf

Mayorga, R., Hurtado, G., \& Benavides, H. (2011). Evidencias de cambio climático en Colombia con base en información estadística. Instituto de Hidrología, Meteorología y Estudios AmbientalesIdeam (Colombia).

Ministerio de Ambiente y Desarrollo Sostenible. (2017). Politica Nacional de Cambio Climático. Ministerio de Ambiente y Desarrollo Sostenible.

Mulligan, M. (2000). Downscaled Climate Change Scenarios for Colombia and Their Hydrological Consequences. Advances in Environmental Monitoring and Modeling, 1(1), 3-35. 
Murillo, L., Pabón, G., Pérez, P., Rojas, M., \& Suárez, R. (2017). Política nacional de cambio climático: documento para tomadores de decisiones. Ministerio de Ambiente y Desarrollo Sostenible. https://repositorio.gestiondelriesgo.gov.co/bitstream/handle/20.500.11762/25548/ Politica-cambio-climatico-MinAmbiente.pdf?sequence=1\&isAllowed $=\mathrm{y}$

Ochoa, M. (2020). Colombia se derrite. Semana. https://sostenibilidad.semana.com/medio-ambiente/articulo/glaciares-de-colombia-se-derriten/48150

Pabón, J. (2012). Cambio climático en Colombia: tendencias en la segunda mitad del siglo XX y escenarios posibles para el siglo XXI. Revista de la Academia Colombiana de Ciencias Exactas, Físicas y Naturales, 36(139), 261-278.

Pabón, J., Cárdenas, I., Kholostyakov, R., Calderón, A., Bernal, N., \& Ruiz, F. (2001). Escenarios climáticos para el siglo XXI sobre el territorio colombiano. Informe Técnico Interno del Instituto de Hidrología, Meteorología y Estudios Ambientales (Ideam).

Posada, C. (2007). La adaptación al cambio climático en Colombia. Revista de ingeniería, (26), 74-80.

Rodríguez, M., \& Mance, H. (2009). Cambio climático: lo que está en juego. Foro Nacional Ambiental.

\section{Bibliografía consultada}

Barton, J. (2009). Adaptación al cambio climático en la planificación de ciudades regiones. Revista de Geografia Norte Grande, (43), 5-30.

Buytaert, W., Sevink, J., \& Cuesta, F. (2014). Cambio climático: la nueva amenaza para los páramos. En W. Buytaert, J. Sevink, \& F. Cuesta (Eds.), Avances en investigación para la conservación de los páramos andinos (pp. 505-526). Condesan.

Instituto de Hidrología, Meteorología y Estudios Ambientales-Ideam. (2010). 2a Comunicación Nacional ante la Convención Marco de las Naciones Unidas sobre Cambio Climático. Ideam.

Ministerio de Ambiente y Desarrollo Sostenible. (2018). AbE Guía de adaptación al cambio climático basada en ecosistemas en Colombia. Ministerio de Ambiente y Desarrollo Sostenible. 